\title{
Discovery of a jet from the single HAe/Be star HD 100546
}

\author{
P. C. Schneider ${ }^{1}$, C. Dougados ${ }^{2}$, E. T. Whelan ${ }^{3}$, J. Eislöffel ${ }^{4}$, H. M. Günther ${ }^{5}$, N. Huélamo ${ }^{6}$, I. Mendigutía ${ }^{6}$, \\ R. D. Oudmaijer ${ }^{7}$, and Tracy L. Beck ${ }^{8}$ \\ 1 Hamburger Sternwarte, Gojenbergsweg 112, 21029 Hamburg, Germany \\ e-mail: astro@pcschneider.eu \\ 2 Univ. Grenoble Alpes, CNRS, IPAG, 38000 Grenoble, France \\ ${ }^{3}$ Maynooth University Department of Experimental Physics, National University of Ireland Maynooth, Maynooth Co. Kildare, \\ Ireland \\ 4 Thüringer Landessternwarte, Sternwarte 5, 07778 Tautenburg, Germany \\ 5 Massachusetts Institute of Technology, Kavli Institute for Astrophysics \& Space Research, 77 Massachusetts Avenue, Cambridge, \\ MA 02139, USA \\ 6 Centro de Astrobiología (CSIC-INTA), Departamento de Astrofísica, ESA-ESAC Campus, PO Box 78, 28692 Villanueva de la \\ Cañada, Madrid, Spain \\ 7 School of Physics and Astronomy, University of Leeds, Leeds LS2 9JT, UK \\ 8 Space Telescope Science Institute, 3700 San Martin Drive, Baltimore, MD 21218, USA
}

Received 1 January 2020 / Accepted 29 April 2020

\begin{abstract}
Young accreting stars drive outflows that collimate into jets, which can be seen hundreds of au from their driving sources. Accretion and outflow activity cease with system age, and it is believed that magneto-centrifugally launched disk winds are critical agents in regulating accretion through the protoplanetary disk. Protostellar jets are well studied in classical T Tauri stars $\left(M_{\star} \lesssim 2 M_{\odot}\right)$, while few nearby $(d \lesssim 150 \mathrm{pc})$ intermediate-mass stars $\left(M_{\star}=2-10 M_{\odot}\right)$, known as Herbig Ae/Be stars, have detected jets. We report VLT/MUSE observations of the Herbig Ae/Be star HD 100546 and the discovery of a protostellar jet. The jet is similar in appearance to jets driven by low-mass stars and compares well with the jet of HD 163296, the only other known optical jet from a nearby Herbig Ae/Be star. We derive a (one-sided) mass-loss rate in the jet of $\log \dot{M}_{\text {jet }} \sim-9.5$ (in $M_{\odot} \mathrm{yr}^{-1}$ ) and a ratio of outflow to accretion of roughly $3 \times 10^{-3}$, which is lower than that of CTTS jets. The discovery of the HD 100546 jet is particularly interesting because the protoplanetary disk around HD 100546 shows a large radial gap, spiral structure, and might host a protoplanetary system. A bar-like structure previously seen in $\mathrm{H} \alpha$ with VLT/SPHERE shares the jet position angle, likely represents the base of the jet, and suggests a jet-launching region within about 2 au. We conclude that the evolution of the disk at radii beyond a few au does not affect the ability of the system to launch jets.
\end{abstract}

Key words. stars: jets - stars: variables: T Tauri, Herbig Ae/Be - circumstellar matter - ISM: jets and outflows stars: individual: HD 100546

\section{Introduction}

Jets are integral to the star formation process, and much of what is understood about astrophysical jets in general comes from studies of young stellar objects (YSOs). Protostellar jets are observed across a wide mass range and in all pre-main sequence evolutionary stages (Frank et al. 2014). Most studies of protostellar jets focused on classical T Tauri stars (CTTSs, class II low-mass YSOs) so that relatively little is known about jets at lower (Whelan 2014) and higher stellar masses, that is, for the intermediate-mass Herbig Ae/Be stars $\left(M_{\star} \sim 2-10 M_{\odot}\right)$. Corcoran \& Ray (1998) reported that the flux ratio between [O $\mathrm{I}$ ] and $\mathrm{H} \alpha$ emission in $\mathrm{HAe} / \mathrm{Be}$ stars follows the same trend as in CTTSs, where a tight connection between accretion and outflow rate has been observed (Cabrit \& Bertout 1992; Hartigan et al. 1995; Günther 2013). Magnetic fields play a crucial role here, and magneto-centrifugal launching is accepted as the likely mechanism for the generation of protostellar jets (Frank et al. 2014), which are launched from the inner disk.
Jet-launching is also thought to affect the planet-forming accretion disk (Baruteau et al. 2014; Dougados 2018).

The focus of this Letter is the single Herbig $\mathrm{Ae} / \mathrm{Be}$ star HD $100546\left(d=109 \pm 4 \mathrm{pc}, M_{\star}=1.9 \pm 0.1 M_{\odot}\right.$; Lindegren et al. 2018; Fairlamb et al. 2015, respectively). Herbig Ae/Be stars typically lack strong stellar magnetic fields (Alecian et al. 2013; Järvinen et al. 2019), like HD 100546 $\left(B_{Z}=89 \pm 26 \mathrm{G}\right.$ compared to $\mathrm{kG}$ fields in CTTSs; Hubrig et al. 2009) so that a transition from magnetically funneled accretion to perhaps boundary layer accretion could be expected for these stars and would likely also affect the jet-driving mechanism (Ellerbroek et al. 2014). HD 100546 is one of the nearest Herbig $\mathrm{Ae} / \mathrm{Be}$ stars and is particularly interesting because there is at least one planetary candidate in the system (e.g., Brittain et al. 2014; Currie et al. 2015; Quanz et al. 2015; Rameau et al. 2017; Cugno et al. 2019). Its protoplanetary disk shows spiral structure (Boccaletti et al. 2013; Follette et al. 2017) and a large inner cavity, which makes HD 100546 a so-called pre-transitional disk system (Brittain et al. 2009; 


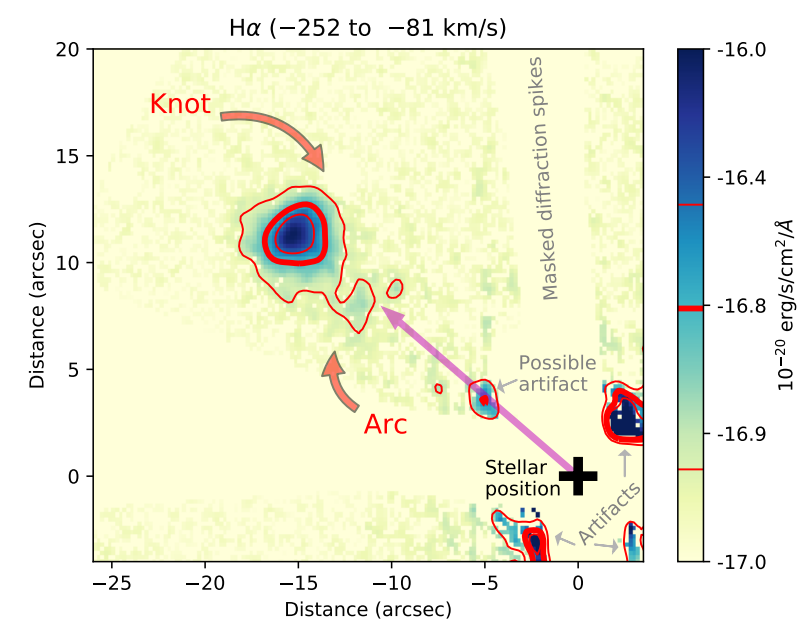

Fig. 1. Majority of the field of view around HD 100546 in H $\alpha$. Stellar emission has been removed, and subtraction residuals are indicated as artifacts.

Mulders et al. 2013; Walsh 2017). HD 100546 had not been reported as driving a jet previous to this work, but asymmetric structures have been reported in the inner disk, such as a bar-like structure in $\mathrm{H} \alpha$ (5-10 au Mendigutía et al. 2017) and a potential disk wind in SO (Booth 2018) and Si III (Grady et al. 2004). We present optical integral field spectroscopic observations of HD 100546, which reveal a jet that is launched perpendicular to the disk major axis in the typical jet lines. Integral field spectroscopy is particularly well suited to the detection and analysis of protostellar jets. Specifically, it allows for the efficient isolation of jet features against contaminating effects such as the Point Spread Function (PSF) wings or background emission and kinemato-morphological studies, and its field of view matches typical sizes of protostellar jets well.

\section{Observations, data reduction, and analysis}

Observations were performed with the European Southern Observatory (ESO) Multi-Unit Spectroscopic Explorer (MUSE; Bacon et al. 2010) located on the ESO Very Large Telescope (VLT). Data for HD 100546 were obtained on 2017 May 4 (seeing 0'.65, airmass 1.4, ESO program 099.C-0.214(A)). The exposure times were consecutively increased during the observing run to balance observing efficiency and saturation effects that were due to the brightness of the central star. Images and spectra presented here are based on data obtained with $t_{\text {exp }}=90 \mathrm{~s}$. The few exposures with (much) shorter integration times provide no significant improvement.

Esoreflex (Freudling et al. 2013) and the MUSE pipeline version 2.2 were used for the data reduction. Because the jet emission is fainter than that of the central star, a PSF subtraction was performed using dedicated python routines, as described in Appendix A. Still, the main jet features are already visible before PSF subtraction (see Fig. A.1). All quoted velocities in this work refer to the stellar rest frame.

\section{Results}

Diffuse emission in typical emission lines associated with protostellar jets is clearly detected in the vicinity of HD 100546; see Fig. 1. This emission consists of an emission knot at a projected distance of $18.4 \operatorname{arcsec}(2005 \mathrm{au})$ from the star (upper left
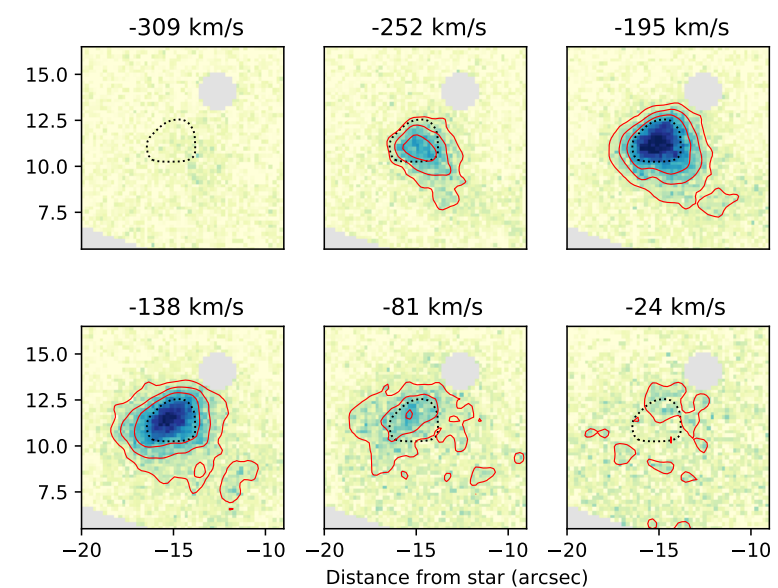

Fig. 2. $\mathrm{H} \alpha$ images of the jet in different velocity channels. To provide a reference between the different velocity channels, the dotted black contour displayed in all panels describes the flux integrated between -252 and $-81 \mathrm{~km} \mathrm{~s}^{-1}$.

quarter in Fig. 1), and its radius is $r \approx 2.5 \operatorname{arcsec}$ where the flux dropped to one-third of the peak value. In addition, there is an arc-like structure closer to the star (below the knot). The line that connects the knot and the arc-like structure points toward HD 100546 and implies a position angle of 53 $(\mathrm{E}$ of $\mathrm{N})$. The channel maps (Fig. 2) show more details of the emission. Specifically, they show that the mean (projected) plasma velocity at the knot is about $-170 \mathrm{~km} \mathrm{~s}^{-1}$, which agrees with that of the arc. In the $-138 \mathrm{~km} \mathrm{~s}^{-1}$ channel, this structure has a banana-like shape: the wings bend backward toward the driving source, which is reminiscent of a typical bow shock. Assuming that the lateral extent of the arc-like structure represents an upper limit on the width of the jet, we find an opening angle of about $10^{\circ}$ for the jet; a similar value is found for the knot.

The pseudo-longslit spectrum extracted along $\mathrm{PA}=53^{\circ}$ reveals emission in the typical jet lines consistently at a distance of 18 arcsec from HD 100546 and at a velocity of about $-170 \mathrm{~km} \mathrm{~s}^{-1}$ (Fig. 3). In addition, line emission is detected all the way down to 6 arcsec from the star, where the signal is lost in the PSF wings of the central star (especially in $\mathrm{H} \alpha$ and [N II] 26583).

The position-velocity diagrams (PVDs) also show the arclike structure (at $14 \operatorname{arcsec}$ ). For the knot at 18.4 arcsec, the velocity of the emission continuously decreases with increasing distance from the star (seen in all lines shown in Fig. 3). In $\mathrm{H} \alpha$, the velocity is $-190 \mathrm{~km} \mathrm{~s}^{-1}$ at a distance of $16^{\prime \prime}$ and just $-110 \mathrm{~km} \mathrm{~s}^{-1}$ at $21^{\prime \prime}$. A similar trend is seen in [N II] $\lambda 6583$ and is likely present in the other lines, although the signal-to-noise ratio $(\mathrm{S} / \mathrm{N})$ is too low to allow firm conclusions for them. With the observed distances and velocities, the dynamical age of the jet is 60 to 90 years, assuming that the jet is launched perpendicular to the disk (with this assumption, the inclination with respect to the sky is either $46^{\circ}$ or $57^{\circ}$, see Mendigutía et al. 2015, 2017, for a discussion of the disk inclination).

The line profiles extracted from the region centered on the $\mathrm{H} \alpha$ peak, specifically from the region enclosed by the thick contour in Fig. 1, are shown in Fig. 4, and the measured line properties are provided in Table 1 (the central star is not subject to a significant amount of extinction so that we did not deredden the measured fluxes; see Fairlamb et al. 2015). The best-fit line shifts are between -160 and $-173 \mathrm{~km} \mathrm{~s}^{-1}$. The wavelength regions around other potential jet lines show no significant emission; an example is the [O III] $\lambda 5007$ line, which is expected to 

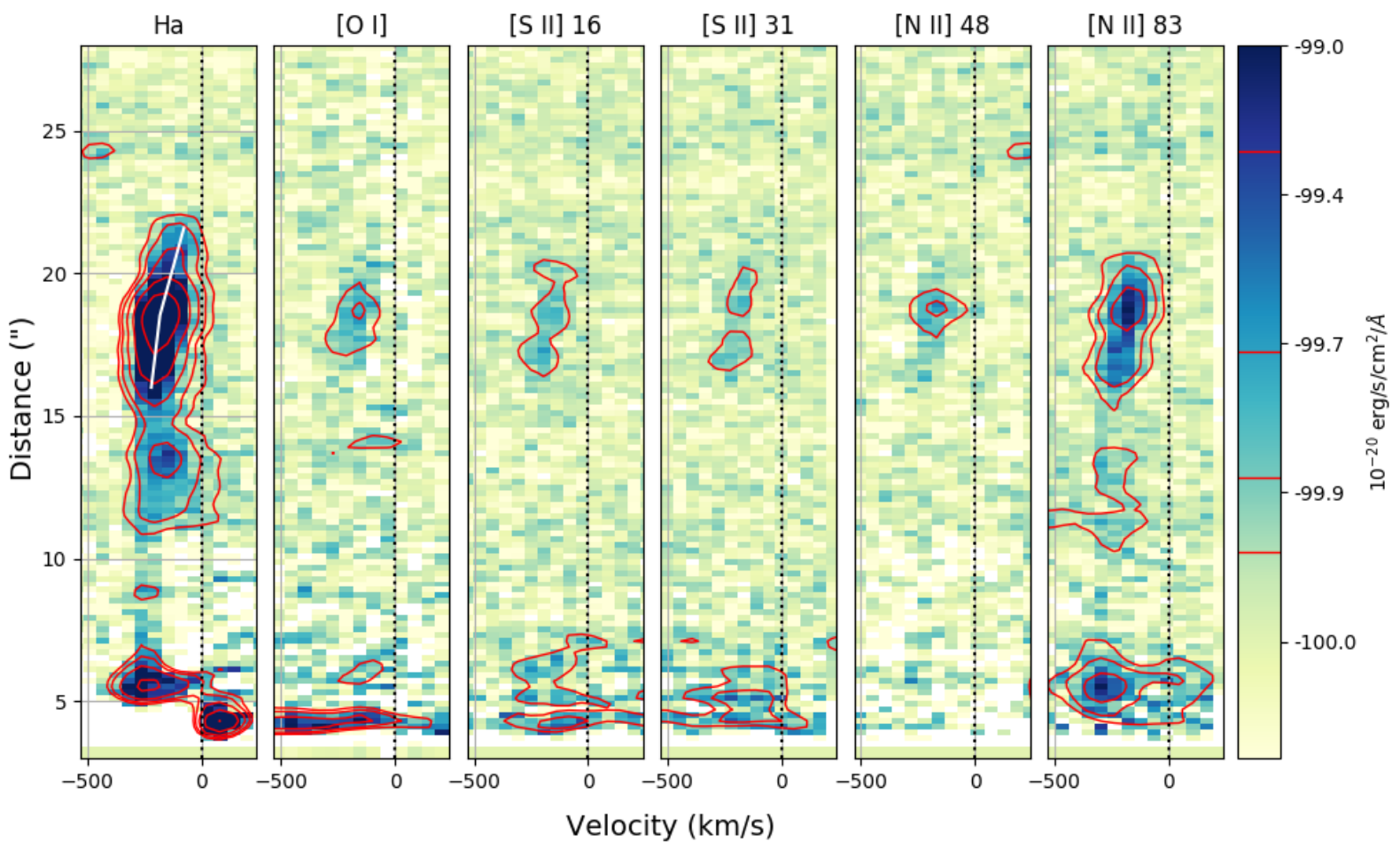

Fig. 3. Position-velocity diagrams of the main jet emission lines. From left to right: H $\alpha$, [O I] $\lambda 6300$, [S II] $\lambda 6716$, [S II] $\lambda 6731$, [N II] $\lambda 6548$, and [N II] 26583 . The white line in the $\mathrm{H} \alpha$ panel indicates the peak velocity.

be strong in highly excited or ionized plasma, that is, for high shock velocities. Other lines also have similar upper limits as [O III]. The line ratio between the two [S II] lines around $6720 \AA$ is density sensitive, and we derive a nominal electron density of $n_{e} \approx 250 \mathrm{~cm}^{-3}$ (the $1 \sigma$ confidence range is $n_{e}<1200 \mathrm{~cm}^{-3}$ from inspection of Fig. 1 in Bacciotti et al. 1995, and the lower value is compatible with the low-density limit of the [S II] ratio so that we cannot formally exclude low densities).

The line ratios, in particular [N $\mathrm{II}] \lambda 6583$ to [S II] $\lambda \lambda 6716,6731$ and [N II] $\lambda 6583$ to [O I] $\lambda 6300$ (see Table 1), suggest shock velocities of $\sim 80 \mathrm{~km} \mathrm{~s}^{-1}$ rather than low-velocity $\left(<50 \mathrm{~km} \mathrm{~s}^{-1}\right)$ shocks, which would for instance have [N II] $\lambda 6583$ to [O I] $\lambda 6300$ of $\lesssim 1.0$ for low jet densities ( $n \leq 1000 \mathrm{~cm}^{-3}$, see Fig. 11 in Hartigan et al. 1994). Interestingly, the ratios of $\mathrm{H} \alpha$ to $\mathrm{H} \beta$ and of [S II] $\lambda 6731$ to $\lambda 6716$ appear more compatible with the Hartigan et al. (1994) models that have a preshock magnetic field of $\gtrsim 100 \mu \mathrm{G}$.

To provide an estimate of the mass fluxes through the jet, we followed Hartigan et al. (1994). Specifically, we used their Eq. (10), that is,

$$
\begin{aligned}
\dot{M}= & 5.95 \times 10^{-8}\left(\frac{10^{3} \mathrm{~cm}^{-3}}{n_{e}}\right)\left(\frac{L_{6300}}{10^{-4} L_{\odot}}\right) \\
& \times\left(\frac{v_{\text {sky }}}{100 \mathrm{~km} \mathrm{~s}^{-1}}\right)\left(\frac{l_{\text {sky }}}{10^{16} \mathrm{~cm}}\right)^{-1} M_{\odot} \mathrm{yr}^{-1},
\end{aligned}
$$

where $L_{6300}, v_{\text {sky }}$, and $l_{\text {sky }}$ are the [O I] line flux, projected jet velocity, and aperture length, respectively.

Our fluxes pertain to an aperture with $\approx 1.5 \operatorname{arcsec}$ radius, so that the projected length on the sky $l_{\text {sky }}$ is $327 \mathrm{au}\left(4.9 \times 10^{15} \mathrm{~cm}\right)$. With a mean projected velocity $v$ of $170 \mathrm{~km} \mathrm{~s}^{-1}$, we find a mass flow of

$\dot{M}_{\text {jet }}=2.8 \times 10^{-10}\left(\frac{250 \mathrm{~cm}^{-3}}{n_{e}}\right) M_{\odot} \mathrm{yr}^{-1}$

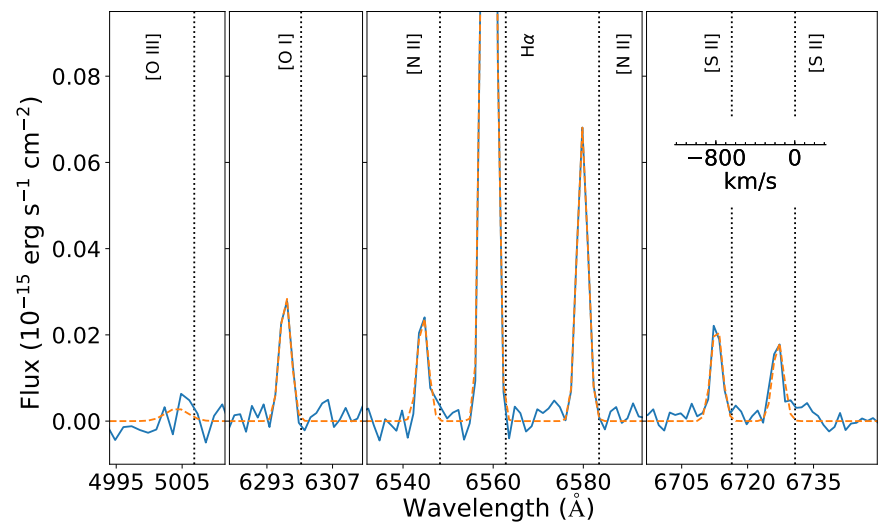

Fig. 4. Selected spectral regions extracted at the knot. The axis scaling is similar in all panels in velocity and flux, i.e., the velocity scale shown in the [S II] panel applies to all four panels equally.

through the blueshifted lobe for $L_{[\mathrm{OI}]}=3.35 \times 10^{-8} L_{\odot}$. Outer streamlines that do not contribute to the shock emission or lower densities would increase the mass loss, while other reasonable numerical constants or methods described in Appendix B provide similar values. We therefore conclude that a mass-loss rate around $\log \dot{M}_{\text {jet }} \sim-9.5$ is very reasonable for the HD 100546 jet. Compared to an accretion rate of $\dot{M}_{\text {acc }} \approx 10^{-7} M_{\odot} \mathrm{yr}^{-1}$ (Mendigutía et al. 2015; Fairlamb et al. 2015), the above values give an outflow-to-accretion rate that is a few times $10^{-3}$, which is well below the typical ratio of $10^{-1}$ (Frank et al. 2014), even when we double this value to account for both jet lobes.

\section{Discussion}

We clearly detect diffuse emission in typical jet lines at distances and blueshifts that are normal for protostellar jets from CTTSs 
Table 1. Line fluxes and ratios.

\begin{tabular}{|c|c|c|c|}
\hline \multicolumn{4}{|c|}{ Line fluxes } \\
\hline Line & $\begin{array}{c}\text { Nominal } \\
\text { wavelength }(\AA)\end{array}$ & $\begin{array}{l}\text { Velocity } \\
\left(\mathrm{km} \mathrm{s}^{-1}\right)\end{array}$ & $\begin{array}{c}\text { Flux } \\
\left(10^{-15} \mathrm{erg} \mathrm{s}^{-1} \mathrm{~cm}^{-2}\right)\end{array}$ \\
\hline [O III] & 5007 & - & $<0.02$ \\
\hline $\mathrm{H} \beta$ & 4861 & $-171 \pm 6$ & $0.296 \pm 0.020$ \\
\hline$[\mathrm{O} \mathrm{I}]$ & 6300 & $-160 \pm 4$ & $0.090 \pm 0.006$ \\
\hline$[\mathrm{N}$ II $]$ & 6548 & $-173 \pm 5$ & $0.075 \pm 0.005$ \\
\hline $\mathrm{H} \alpha$ & 6563 & $-171 \pm 1$ & $0.968 \pm 0.006$ \\
\hline$[\mathrm{N}$ II $]$ & 6583 & $-168 \pm 2$ & $0.206 \pm 0.004$ \\
\hline$[\mathrm{S}$ II $]$ & 6716 & $-155 \pm 18$ & $0.082 \pm 0.017$ \\
\hline$[\mathrm{S}$ II $]$ & 6731 & $-162 \pm 16$ & $0.069 \pm 0.017$ \\
\hline \multicolumn{4}{|c|}{$\begin{array}{l}\text { Line ratios } \\
\end{array}$} \\
\hline Numerator & Denominator & Ratio & log ratio \\
\hline [S II] 6716 & [S II] 6731 & $1.19 \pm 0.38$ & 0.07 \\
\hline [N II] 6583 & [O I $] 6300$ & $2.29 \pm 0.16$ & 0.36 \\
\hline [S II] 6731 & $\mathrm{H} \alpha 6563$ & $0.07 \pm 0.02$ & -1.15 \\
\hline Н $\alpha 6563$ & $\mathrm{H} \beta 4861$ & $3.35 \pm 0.23$ & 0.53 \\
\hline [N II] 6583 & [S II] 6716+6731 & $1.36 \pm 0.22$ & 0.13 \\
\hline [O I] 6300 & $\mathrm{H} \alpha 6563$ & $0.093 \pm 0.006$ & -1.03 \\
\hline
\end{tabular}

and HAe/Be stars. Furthermore, the PA of the jet is perpendicular to the disk major axis $\left(\mathrm{PA} \approx 137^{\circ}\right.$; Mendigutía et al. 2017), and a jet launched from the HD 100546 disk would be blueshifted at the PA of the diffuse emission given the disk inclination $\left(i \approx 44^{\circ}\right.$ for the inner and outer disk, Mendigutía et al. 2015). We therefore interpret the diffuse emission as the protostellar jet from HD 100546. This jet emission went unnoticed in previous data of HD 100546 probably because the central star is very bright. This brightness requires short integration times to avoid saturation effects. However, jet features will remain hidden in the noise with short exposures. For our observations, we deliberately overexposed the star to increase the detectability of jet features. Another possibility to explain the nondetection of the jet in previous data is the sky coverage in spectroscopic data was not good enough.

The most prominent features of the MUSE data are the knotand arc-like features. A collimated jet is not immediately recognizable in the MUSE data, which we attribute to the relative brightness of the bow shock and the jet body: the jet emission is also far weaker than the bow shock in the prototypical HH 1/2 system (Hartigan et al. 2011). In addition, PSF spillover hinders the detection of jet emission close to the driving source. Still, the $\mathrm{H} \alpha$ and [N II] $\lambda 6583$ PVDs reveal that jet emission can be traced back to HD 100546 at essentially the same velocity as farther out, so that we are confident that HD 100546 is the driving source of the jet.

We note that the nondetection of the counter jet is not surprising: one jet lobe is typically brighter and sometimes also less collimated (Hirth et al. 1994), so that its emission is likely lost in the region close to the driving source. In the specific case of the HD 100546 MUSE data, the counter jet should be visible if it were of comparable brightness as the forward jet, within the MUSE field of view (approximately 27 arcsec or $\sim 3000$ au in the jet direction, see Fig. A.1), and beyond the outer radius of the disk (260 au and 400 au for the dust and gas disk, respectively; see Pinilla et al. 2015, for a discussion of the HD 100546 disk sizes).

\subsection{Jet properties and relation to disk structure}

The jet axis aligns well with the bar-like feature (PA $45^{\circ}$ ) seen in polarized $\mathrm{H} \alpha$ and continuum light detected by
Mendigutía et al. (2017), which can be traced down to about 2 au from the position of HD 100546; this is about the size of the inner disk. Mendigutía et al. (2017) previously remarked on the similarity of this feature to a bipolar outflow. We are therefore tempted to associate the bar with the base of the jet. Mendigutía et al. (2017) discarded the jet explanation because no jet was known for HD 100546 and no blueshifted excess emission was seen in [O I] $\lambda 6300$ (Acke \& van den Ancker 2006), which is a typically feature in jet-driving CTTSs. The CO emission of HD 100546 also appears to require nonKeplerian disk emission in the bar region (Walsh 2017), which has been interpreted in terms of a warped disk and radial flows, but may instead be associated with the outflow detected here.

The association of the bar-like feature with the base of the jet is particularly interesting because it provides a strong constraint for the launching region $(\lesssim 2 \mathrm{au})$. Such a small launching region is generally consistent with other observational constraints such as jet rotation measurements and their upper limits, which locate the launching region within about 1 au for most CTTS jets (Ferreira et al. 2006; Coffey et al. 2015). This also agrees with the interpretation of high-resolution spectra of CTTSs that suggest a jet origin within a few au from the star for all kinematic components (low-velocity broad or narrow components and high-velocity components; Fang et al. 2018).

A small launch radius is also compatible with jet models: they postulate that outflows that collimate into jets are launched within $\sim 10$ au from the driving source (Pudritz et al. 2007) such as magnetospheric ejections (Zanni \& Ferreira 2013; Romanova \& Owocki 2016) and some disk-wind models in which the highest mass-loss occurs within about 1 au (for CTTSs, see Nolan et al. 2017). On the other hand, the significant mass-loss out to radii of 10-20 au present in some magnetohydrodynamical simulations of full disks (e.g., Bai 2014; Gressel 2015; Béthune et al. 2017) is neither seen in our MUSE nor in the SPHERE data of HD 100546. Outflows launched from larger radial distances are expected to be slower, and Fang et al. (2018) suggested that they might be invisible in the classical jet lines because their temperature is low $(<5000 \mathrm{~K})$. Specifically for HD 100546, however, slower outflow components would still be fast enough to shock heat material sufficiently so that it radiates in the typical jet lines. The lack of slow jet components in HD 100546 might therefore also be rooted in the disk gap (including gas depletion, see van der Plas et al. 2009) because this would also lower the mass-loss rate compared to the rate in full disks. Without angular momentum removal by the jet, radial flows are suppressed and no or only little material would be transported through the gap, so that stellar accretion may be limited to the material in the inner disk. In this scenario, HD 100546 would be about to cease accretion within a few centuries (Mendigutía et al. 2015; Pineda et al. 2019, who reported a total inner disk mass of $5 \times 10^{-5} M_{\odot}$ ).

\subsection{Comparison to other jets}

The HD 100546 jet shows many features seen in other protostellar jets. First, the emission is concentrated into rather discrete entities (knots). A (semi-) regular chain of emission knots (as seen, e.g., for HD 163296) is not detected in the MUSE data, however. This might be related to detection thresholds because additional emission knots might remain undetected at larger distances because the surface brightness might be too low, and closer to the star, the contrast against the PSF wings might be too low. 
Second, the projected and deprojected velocities of $\approx 170 \mathrm{~km} \mathrm{~s}^{-1}$ and $230 \mathrm{~km} \mathrm{~s}^{-1}$ fall well within the velocity range of most atomic protostellar jets (Eislöeffel \& Mundt 1998; Frank et al. 2014, the deprojected velocity assumes that the jet is launched perpendicular to the disk). The jets of HD 163296 and $\mathrm{LkH} \alpha 233$, driven by similarly massive central stars, show very similar velocities (Ellerbroek et al. 2014; Melnikov et al. 2008). Compared to jets in Orion and Carina (O'Dell \& Doi 2003; Reiter et al. 2017), the HD 100546 jet is fast, but it is reasonable to assume that the apparent deceleration of the HD 100546 jet between about 18 and 21 arcsec (Fig. 3) would lead to similar velocities if it were observed at larger distances, as are most of the Orion and Carina jets.

Third, the density $n_{e}$ compares well with that of HD 163296, but is lower than that of other jets, such as the jet of $\mathrm{LkH} \alpha 233$. Still, densities derived for other jets are within about one order of magnitude at similar distances because the highest densities are typically found close to the driving source. We do not have a significant detection or density diagnostics for the HD 100546 jet at short distances.

The mass-loss rate is on the very low end compared to the typical outflow rates of $10^{-7}$ to $10^{-9} M_{\odot} \mathrm{yr}^{-1}$ measured for CTTSs (Frank et al. 2014), and new observations of HD 100546 are required to determine whether this also holds for the jet close to the launch site. In particular, the measured outflow-toaccretion rate is lower $\left(3 \times 10^{-3}\right.$ for the blueshifted lobe $)$ than typical ratios, which are usually between $10^{-2}$ and $10^{-1}$ (see Frank et al. 2014), also for the brighter knots in the HD 163296 jet and likely for $\mathrm{LkH} \alpha 233^{1}$. Taken together, the HD 100546 jet in the atomic lines traced by the MUSE data appears to be less powerful than other jets, both in absolute terms and in relation to the stellar accretion rate.

\section{Conclusions}

The presence of a collimated jet in a nonmagnetic or weakly magnetic relatively massive and evolved star with a small inner disk provides immediate constraints on the jet-launching mechanism. First, the similarity in appearance between the HD 100546 jet and CTTS and other HAe/Be jets suggests a similar launching mechanism, that is, an origin within the innermost au from the central star. Mendigutía et al. (2015) found that HD 100546 likely accretes magnetospherically (in contrast to boundary layer accretion), and the weak stellar magnetic field ( $\lesssim 100 \mathrm{G})$ may be sufficient to make HD 100546 a scaled-up version of a CTTS, including its jet. Second, regardless of what causes the formation of disk gaps or spirals, the jet-launching mechanism is left intact. Finally, planet formation and jets are not mutually exclusive if any of the planetary candidates in HD 100546 is real.

Herbig $\mathrm{Ae} / \mathrm{Be}$ stars are by definition relatively evolved objects compared to many CTTSs, so that the accretion as well as the outflow rate are expected to have decreased compared to younger systems; specifically, HD 100546 has a quoted age of $4.8_{-0.2}^{+2.0} \mathrm{Myr}$ (Wichittanakom et al. 2020; Acke \& van den Ancker 2006), which is older than the typical CTTS age of 1-2 Myr. We may speculate that the low outflow rates at continued high accretion rates imply that mass-outflow rates decline before the accretion drops. Fang et al. (2018) found that the occurrence frequency of the high-velocity component in typical jet lines is significantly lower in systems with transi-

\footnotetext{
1 Precise mass accretion rates are not available for $\mathrm{LkH} \alpha 233$, but the estimated outflow rate of $10^{-8} M_{\odot} \mathrm{yr}^{-1}$ would result in $M_{\text {out }} / M_{\text {acc }} \gtrsim 0.1$ for typical stellar accretion rates.
}

tional disks than in full disks, while stellar accretion rates may be very similar (Manara et al. 2014). Within this picture, the low mass-loss rate of the HD 100546 jet may indicate an evolutionary sequence with jet mass-loss rates that decrease first and stellar accretion rates that decrease only later, in the phase when the residual material in the inner disk is consumed and the supply of disk material from outer disk radii is reduced or absent. If the disappearance of the jet causally changes the disk, for example, by reducing radial transport through the disk, or if jet activity ceases as a result of other processes such as gap formation needs to be investigated in future work.

Acknowledgements. PCS gratefully acknowledges support by DLR 50 OR 1901. HMG acknowledges support by NASA-HST-GO-12315.01. NH has been partially funded by the Spanish State Research Agency (AEI) Project No. ESP2017-87676-C5-1-R and No. MDM-2017-0737 Unidad de Excelencia 'María de Maeztu'- Centro de Astrobiología (INTA-CSIC). Support for this work was provided by the National Aeronautics and Space Administration through Chandra Award Number GO5-16014X issued by the Chandra X-ray Observatory Center, which is operated by the Smithsonian Astrophysical Observatory for and on behalf of the National Aeronautics Space Administration under contract NAS8-03060. Based on observations collected at the European Southern Observatory under ESO programme 099.C-0214(A).

\section{References}

Acke, B., \& van den Ancker, M. E. 2006, A\&A, 449, 267

Alecian, E., Wade, G. A., Catala, C., et al. 2013, MNRAS, 429, 1001

Bacciotti, F., Chiuderi, C., \& Oliva, E. 1995, A\&A, 296, 185

Bacon, R., Accardo, M., Adjali, L., et al. 2010, Ground-based and Airborne Instrumentation for Astronomy III, 773508

Bai, X.-N. 2014, ApJ, 791, 137

Baruteau, C., Crida, A., Paardekooper, S.-J., et al. 2014, Protostars and Planets VI, 667

Béthune, W., Lesur, G., \& Ferreira, J. 2017, A\&A, 600, A75

Boccaletti, A., Pantin, E., Lagrange, A.-M., et al. 2013, A\&A, 560, A20

Booth, A. S., Walsh, C., Kama, M., et al. 2018, A\&A, 611, A16

Brittain, S. D., Najita, J. R., \& Carr, J. S. 2009, ApJ, 702, 85

Brittain, S. D., Carr, J. S., Najita, J. R., et al. 2014, ApJ, 791, 136

Cabrit, S., \& Bertout, C. 1992, A\&A, 261, 274

Coffey, D., Dougados, C., Cabrit, S., et al. 2015, ApJ, 804, 2

Corcoran, M., \& Ray, T. P. 1998, A\&A, 331, 147

Cugno, G., Quanz, S. P., Hunziker, S., et al. 2019, A\&A, 622, A156

Currie, T., Cloutier, R., Brittain, S., et al. 2015, ApJ, 814, 27

Dougados, C. 2018, Take a Closer Look, 14

Eislöeffel, J., \& Mundt, R. 1998, AJ, 115, 1554

Ellerbroek, L. E., Podio, L., Dougados, C., et al. 2014, A\&A, 563, A87

Fairlamb, J., Oudmaijer, R. D., Mendigutía, I., et al. 2015, MNRAS, 453, 976

Fang, M., Pascucci, L., Edwards, S., et al. 2018, ApJ, 868, 28

Ferreira, J., Dougados, C., \& Cabrit, S. 2006, A\&A, 453, 785

Follette, K. B., Rameau, J., Dong, R., et al. 2017, ApJ, 153, 264

Frank, A., Ray, T. P., Cabrit, S., et al. 2014, Protostars and Planets VI, 451

Freudling, W., Romaniello, M., Bramich, D. M., et al. 2013, A\&A, 559, A96

Grady, C. A., Woodgate, B., Heap, S. R., et al. 2004, ApJ, 620, 470

Gressel, O., Turner, N. J., Nelson, R. P., et al. 2015, ApJ, 801, 84

Günther, H. M. 2013, Astron. Nachr., 334, 67

Hartigan, P., Morese, J.A., \& Raymond, J. 1994, ApJ, 125, 436

Hartigan, P., Edwards, S., \& Ghandour, L. 1995, ApJ, 452, 736

Hartigan, P., Frank, A., Foster, J. M., et al. 2011, ApJ, 735, 29

Hirth, G. A., Mundt, R., Solf, J., et al. 1994, ApJ, 427, 99

Hubrig, S., Stelzer, B., Schöller, M., et al. 2009, A\&A, 502, 283

Järvinen, S. P., Carroll, T. A., Hubrig, S., et al. 2019, MNRAS, 489, 886

Lindegren, L., Hernández, J., Bombrun, A., et al. 2018, A\&A, 616, A2

Manara, C. F., Testi, L., Natta, A., et al. 2014, A\&A, 568, A18

Mendigutía, I., de Wit, W. J., Oudmaijer, R. D., et al. 2015, MNRAS, 453, 2126

Mendigutía, I., Oudmaijer, R. D., Garufi, A., et al. 2017, A\&A, 608, A104

Melnikov, S., Woitas, J., Eislöffel, J., et al. 2008, A\&A, 483, 199

Mulders, G. D., Paardekooper, S.-J., Panić, O., et al. 2013, A\&A, 557, A68

Nolan, C. A., Salmeron, R., Federrath, C., et al. 2017, MNRAS, 471, 1488

O’Dell, C. R., \& Doi, T. 2003, AJ, 125, 277

Pineda, J. E., Szulágyi, J., Quanz, S. P., et al. 2019, ApJ, 871, 48

Pinilla, P., Birnstiel, T., \& Walsh, C. 2015, A\&A, 580, A105

Pudritz, R. E., Ouyed, R., Fendt, Ch., \& Brandenburg, A. 2007, Protostars and Planets V, 277 
Quanz, S., Amara, A., Meyer, M. R., et al. 2015, ApJ, 807, 64

Rameau, J., Follette, K. B., Pueyo, L., et al. 2017, AJ, 153, 244

Romanova, M., \& Owocki, S. P. 2016, The Strongest Magnetic Fields in the Universe, 347

Reiter, M., Kiminki, M. M., Smith, N., \& Bally, J. 2017, MNRAS, 470, 4671

van der Plas, G., van den Ancker, M. E., \& Acke, B. 2009, A\&A, 500, 1137

Walsh, C., Daley, C., Facchini, S., et al. 2017, A\&A, 607, A114

Whelan, E. T. 2014, Astron. Nachr., 335, 537

Wichittanakom, C., Oudmaijer, R. D., Fairlamb, J. R., et al. 2020, MNRAS, 293 , 234

Zanni, C., \& Ferreira, J. 2013, A\&A, 550, A99

\section{Appendix A: Data reduction details}

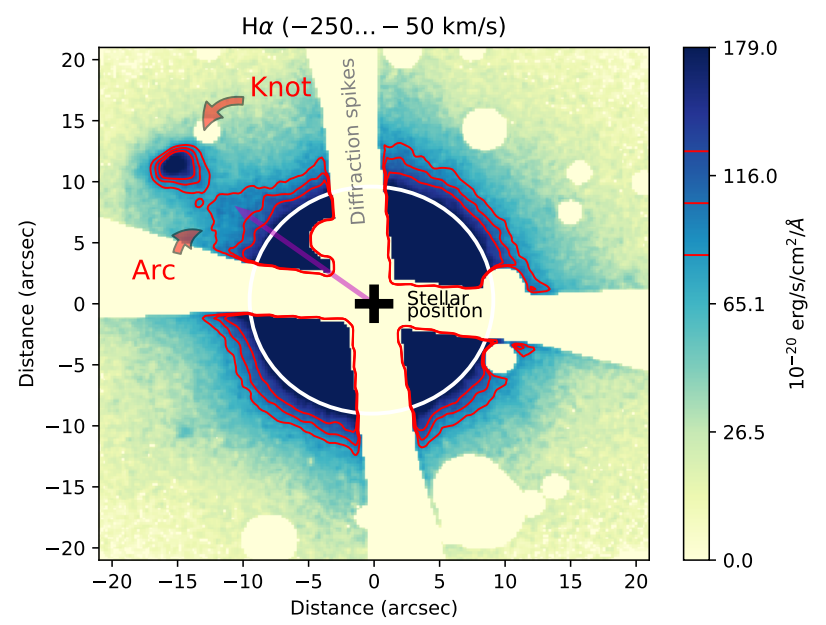

Fig. A.1. Raw image of HD 100546. Most of the field of view around HD 100546 in $\mathrm{H} \alpha$ before the stellar emission was removed. The knot and arc are already visible before PSF subtraction. The white circle shows the symmetry of the stellar PSF.

Data reduction generally followed standard procedures. However, the immediate region around the central star is heavily affected by saturation effects. The particular sub-IFUs of the 24 individual sub-IFUs of MUSE, which are most strongly affected by the central star, were discarded during further data reduction. Each of the 24 sub-IFUs covers a particular patch on the sky, and discarding one to two sub-IFUs creates empty sky patches for each exposure. Full sky coverage ${ }^{2}$ is then obtained by rotating the detector by $22.5 \mathrm{deg}$ between individual exposures, as is generally recommended to improve spatial homogeneity, and then combing the individual exposures.

Point-spread function subtraction is performed by considering the PSF as a combination of three radially symmetric exponential functions. Moffat functions, typically used to describe the geometrical form of a PSF, were found to perform less well in the outer parts of the PSF $(d \gtrsim 5$ arcsec). PSF fitting (or rather the PSF wing fitting) was performed individually for several wavelength regions containing one to several relevant jet emission lines. During the fitting procedure, background stars, the diffraction spikes, and the sky patches containing jet emission were discarded. For each relevant emission line, the PSF fit was then performed to a cube restricted to wavelengths around the line centroid (approximately $30 \AA$, excluding the -400 to $+100 \mathrm{~km} \mathrm{~s}^{-1}$ range around the nominal wavelength). The fit assumes that the PSF has the same wavelength dependence as the stellar spectrum extracted from an unsaturated region close to the stellar position to minimize the number of free parameters. The PSF model ensures spatial and spectral smoothness, requires only a small number of free parameters, and was found to leave systematic PSF subtraction residuals only very close to the star and the diffraction spikes (see Fig. A.1).

\section{Appendix B: Alternative mass-loss estimate}

When we used Eq. (A8) in Hartigan et al. (1995) or the numerical constants used by Fang et al. (2018), we obtained similar values (differences $\lesssim 15 \%$ ) as those described in the main text. Also, the alternative method to estimate the mass-loss rate based on area $A$, velocity $v$, and density estimates $(n)$ results in comparable values:

$\dot{M}=\rho v A=\mu m_{H} n v A$,

where $\rho=\mu m_{H} n$ is the mass density with the mean molecular weight $\mu=1.24$, the mass of the hydrogen atom $m_{H}$, and the number density $n$. To estimate $n$ from $n_{e}$ derived from the [S II] doublet, Hartigan et al. (1994) recommend to use

$n=\frac{n_{e}}{\sqrt{\langle C\rangle}\langle I\rangle}$,

with the ionization $\langle I\rangle$ fraction and the mean compression factor $\langle C\rangle$.

The HD 100546 jet appears to be of low density $\left(n_{e} \sim\right.$ $\left.250 \mathrm{~cm}^{-3}\right)$ and to have a high $\left(\sim 80 \mathrm{~km} \mathrm{~s}^{-1}\right)$ shock velocity. Therefore, $\log \langle C\rangle \sim 2$ (cf. Fig. 17 in Hartigan et al. 1994), while $\langle I\rangle$ may be around 0.5. Equation (B.2) therefore indicates that $n \sim 50 \mathrm{~cm}^{-3}$ (for $n_{e}=250 \mathrm{~cm}^{-3}$ ), and Eq. (B.1) gives $\dot{M} \sim 7 \times 10^{-10} M_{\odot} \mathrm{yr}^{-1}$. This is reasonably close to the value provided in Sect. 3 .

2 Except for about 2 arcsec around the central star. 